\title{
Vitamin $E$ status has an impact on plasma $n-3$ fatty acid proportion in a healthy adult Irish population
}

\author{
Y. Zhao, F.J. Monahan, B.A. McNulty, A.P. Nugent, K.F. Li, L. Brennan and E.R. Gibney \\ UCD Institute of Food and Health, University College Dublin, Ireland
}

Many of the health benefits of vitamin E intake are believed to derive from its antioxidant properties ${ }^{(1)}$, among them the prevention of oxidation of $\mathrm{PUFA}^{(2)}$. Previous animal studies have demonstrated the importance of dietary vitamin E in protecting circulating PUFA $^{(3,4)}$; however, to our knowledge this has not been investigated in humans.

This study aimed to determine the relationship between habitual vitamin E intake and plasma $\alpha$-tocopherol concentrations on plasma fatty acid proportion, in a nationally representative sample. Dietary intake data from the National Adult Nutrition Survey (NANS) ${ }^{(5)}$ were used; valid reporters who did not consume supplements containing PUFA were included in the study $(\mathrm{n}=601)$. Plasma $\alpha$-tocopherol concentrations were measured by HPLC and plasma fatty acids were extracted and quantified using GC-MS. Participants were divided into vitamin E intake quartiles and plasma $\alpha$-tocopherol quartiles and differences in plasma fatty acid proportion were explored by general linear regression using SPSS.

Across both vitamin E intake and plasma $\alpha$-tocopherol quartiles, PUFA intake significantly increased. Following adjustment for

\begin{tabular}{|c|c|c|c|c|c|c|c|c|c|}
\hline & \multicolumn{8}{|c|}{ Vitamin $\mathrm{E}$ intake quartiles } & \multirow[b]{3}{*}{ GLM* } \\
\hline & \multicolumn{2}{|c|}{$1(\mathrm{n}=150)$} & \multicolumn{2}{|c|}{$2(\mathrm{n}=150)$} & \multicolumn{2}{|c|}{$\underline{3(n=151)}$} & \multicolumn{2}{|c|}{$4(n=150)$} & \\
\hline & Mean & SD & Mean & SD & Mean & SD & Mean & SD & \\
\hline Vitamin E mean daily intake (mg/d) & $6 \cdot 0$ & $1 \cdot 1$ & $9 \cdot 0$ & $0 \cdot 7$ & $11 \cdot 9$ & $1 \cdot 0$ & $20 \cdot 5$ & $8 \cdot 5$ & \\
\hline PUFA $(\%)$ & $42 \cdot 1$ & $4 \cdot 5$ & $41 \cdot 8$ & $4 \cdot 4$ & $42 \cdot 7$ & $4 \cdot 0$ & $43 \cdot 0$ & 4.4 & $0 \cdot 412$ \\
\hline n-3 PUFA $(\%)$ & $5 \cdot 0^{\mathrm{c}}$ & 1.2 & $5 \cdot 2^{\mathrm{bc}}$ & 1.4 & $5 \cdot 6^{\mathrm{ab}}$ & $2 \cdot 0$ & $5 \cdot 8^{\mathrm{a}}$ & $2 \cdot 0$ & $\mathrm{P}<0.001$ \\
\hline$\alpha$-Linolenic acid (C18:3n3) (\%) & $0 \cdot 80$ & $0 \cdot 31$ & $0 \cdot 82$ & $0 \cdot 26$ & $0 \cdot 86$ & $0 \cdot 22$ & $0 \cdot 88$ & $0 \cdot 28$ & $0 \cdot 282$ \\
\hline Eicosatetraenoic acid (C20:4n3) (\%) & $0 \cdot 20$ & $0 \cdot 20$ & $0 \cdot 19$ & $0 \cdot 08$ & $0 \cdot 28$ & $1 \cdot 13$ & $0 \cdot 18$ & 0.09 & $0 \cdot 828$ \\
\hline Eicosapentaenoic acid (C20:5n3) (\%) & $1 \cdot 08^{\mathrm{b}}$ & 0.46 & $1 \cdot 20^{\mathrm{ab}}$ & $0 \cdot 63$ & $1.29^{\mathrm{ab}}$ & 0.69 & $1.43^{\mathrm{a}}$ & 0.94 & $0 \cdot 011$ \\
\hline Docosapentaenoic acid (C22:5n3) (\%) & 0.79 & $0 \cdot 19$ & 0.79 & $0 \cdot 19$ & $0 \cdot 83$ & $0 \cdot 20$ & $0 \cdot 83$ & $0 \cdot 20$ & $0 \cdot 110$ \\
\hline Docosahexaenoic acid (C22:6n3) (\%) & $2 \cdot 10^{\mathrm{c}}$ & 0.68 & $2 \cdot 21^{\mathrm{bc}}$ & $0 \cdot 78$ & $2 \cdot 37^{\mathrm{ab}}$ & 0.92 & $2 \cdot 47^{\mathrm{a}}$ & 1.03 & 0.002 \\
\hline n-6 PUFA (\%) & $36 \cdot 9$ & $4 \cdot 4$ & $36 \cdot 5$ & $4 \cdot 4$ & $36 \cdot 9$ & $3 \cdot 9$ & $37 \cdot 2$ & $4 \cdot 3$ & 0.799 \\
\hline Linoleic acid (C18:2n6c) (\%) & $26 \cdot 0$ & $4 \cdot 1$ & $25 \cdot 7$ & $3 \cdot 9$ & $26 \cdot 5$ & $3 \cdot 5$ & $26 \cdot 6$ & $4 \cdot 0$ & 0.767 \\
\hline$\gamma$-Linolenic (C18:3n6) (\%) & $0 \cdot 57$ & $0 \cdot 19$ & $0 \cdot 56$ & $0 \cdot 18$ & $0 \cdot 54$ & $0 \cdot 19$ & $0 \cdot 56$ & $0 \cdot 20$ & $0 \cdot 534$ \\
\hline Eicosadienoic acid (C20:2n6) (\%) & $0 \cdot 38$ & $0 \cdot 22$ & $0 \cdot 39$ & $0 \cdot 18$ & $0 \cdot 39$ & $0 \cdot 19$ & $0 \cdot 39$ & $0 \cdot 15$ & $0 \cdot 537$ \\
\hline Eicosatrienoic acid (C20:3n6) (\%) & $2 \cdot 09^{\mathrm{a}}$ & 0.44 & $2 \cdot 12^{\mathrm{a}}$ & 0.53 & $2 \cdot 04^{\mathrm{ab}}$ & 0.45 & $1.99^{\mathrm{b}}$ & 0.47 & 0.023 \\
\hline Arachidonoic acid (C20:4n6) (\%) & $7 \cdot 74$ & 1.87 & $7 \cdot 53$ & $2 \cdot 05$ & $7 \cdot 52$ & 1.67 & $7 \cdot 44$ & $1 \cdot 62$ & $0 \cdot 873$ \\
\hline Docosatetraenoic acid (C22:4n6) (\%) & $0 \cdot 27^{\mathrm{a}}$ & $0 \cdot 10$ & $0 \cdot 24^{\mathrm{b}}$ & 0.08 & $0 \cdot 24^{\mathrm{ab}}$ & $0 \cdot 10$ & $0 \cdot 23^{\mathrm{ab}}$ & 0.08 & $0 \cdot 043$ \\
\hline$n-3 / n-6$ & $0 \cdot 14^{\mathrm{b}}$ & $0 \cdot 04$ & $0 \cdot 14^{\mathrm{ab}}$ & $0 \cdot 04$ & $0 \cdot 16^{\mathrm{a}}$ & $0 \cdot 07$ & $0 \cdot 16^{\mathrm{a}}$ & $0 \cdot 06$ & $0 \cdot 001$ \\
\hline
\end{tabular}

* general linear model univariate analysis, adjusted for gender, vitamin E containing supplement use, total energy and corresponding PUFA intake as percentage of total energy $(\% \mathrm{TE})$ intake, was carried out to determine significance with log transformed value; different superscript letters denote significant differences across vitamin $\mathrm{E}$ intake quartiles $(\mathrm{P}<0.05)$

PUFA intake, vitamin $\mathrm{E}$ intake showed positive correlations with plasma n-3 PUFA $(\mathrm{P}<0 \cdot 001), \mathrm{EPA}(\mathrm{P}=0 \cdot 011)$ and DHA $(\mathrm{P}=$ $0.002)$ proportion and plasma $n-3 / n-6$ ratio $(P=0.001)$ and a negative correlation with plasma eicosatrienoic acid $(P=0.023)$ proportion. When plasma $\alpha$-tocopherol was examined, only plasma $\alpha$-linolenic acid (ALA) proportion significantly increased with increasing plasma $\alpha$-tocopherol (data not shown). These results suggest that circulating plasma n-3 PUFA (EPA and DHA) may be protected by vitamin E intake.

The work was jointly funded by the Department of Agriculture, Food and Marine under the Food for Health Research Initiative; Ms Zhao is in receipt of a PhD student from the China Scholarship Council 2011-2015.

1. Cardenas E \& Ghosh R (2013) Biochem Pharmacol 86, 845-852.

2. Weber P, Bendich A \& Machlin LJ (1997) Nutrition 13, 450-460.

3. Lebold KM \& Traber MG (2013) Free Radic Biol Med 66, 13-19.

4. Guo Q, Richert BT, Burgess JR, et al. (2006) J Anim Sci 84, 3089-3099.

5. Irish Universities Nutrition Alliance (IUNA). National Adult Nutrition Survey Summary Report. Food Safety Promotion Board, Abbey Court, lower Abbet Street, Dublin 1: Irish Universities Nutrition Alliance, 2011. 